\title{
A WEB PORTAL FOR OPEN-SOURCE SYNCHRONOUS DISTANCE EDUCATION
}

\author{
J. Mark Pullen and Priscilla M. McAndrews \\ Networking and Simulation Laboratory, C3I Center \\ George Mason University \\ Fairfax, VA 22030 \\ mpullen@gmu.edu,pmcandre@gmu.edu
}

\begin{abstract}
Network EducationWare (NEW) is an integrated collection of open-source software for synchronous Internet communication, where a class is simultaneously taught to local students and distributed over the Internet. In order to scale use of NEW to multiple courses, we have developed a Web portal that provides coordinated access to each major function. We describe the way this portal works with the NEW client and server software to reduce effort and enhance effectiveness of online course management. The resulting system provides a simple, low-cost means of tending teaching and learning over the Internet. The portal webpages and other NEW software are available at no cost for any academic activity.
\end{abstract}

\section{Keywords}

Web portal, synchronous distance education, multimedia

\section{Introduction}

The Internet is widely used for asynchronous distance education, consisting mostly of remote access to Webbased course materials [1]. Certainly, this use of remote access saves a lot of travel to the library and also saves many trees from being sacrificed for paper. In cases where it is used to provide a full course, rather than simply augmenting an instructor-taught course, it provides a faster means of delivery for that earlier form of distance education, the correspondence course. However, we observe that, over many generations of schooling, the academic enterprise has worked out an approach to teaching that, for the average student, is most effective at promoting learning. We refer to the traditional mode where the instructor serves as mentor and interpreter of course materials and a regular schedule of assignments keeps students engaged in learning. The Internet does not invalidate this conclusion. On the contrary, just as the Internet has made the correspondence course more accessible and flexible, synchronous distance education creates an ability to deliver traditional, instructorpresented classes to students who find that distance makes classroom attendance too expensive or simply impractical.

Until recently, synchronous distance education was delivered primarily by television. Today the Internet, combined with the personal computer, offers a means of electronic delivery that can be more effective than television, both educationally and in terms of cost to deliver. While many faculty members are conditioned by "television teaching" to assume that video plus audio is the most effective means of delivery, our experience shows that in many cases audiographics (the combination of audio, prepared graphics, and dynamic graphic annotation) is more effective than video [2]. Moreover, audiographics requires much less network capacity than video and therefore reduces the cost of synchronous course delivery [3][4][5]. Therefore, as reported in [6][7][8], we have developed a software system for synchronous distance education by integrating open source software from other groups with our own supplemental software.

Managing courses that cater to synchronous Internet students has provided a considerable challenge. The first realization we faced was that simply providing a lecture to a distant student does not provide an entire educational solution. As presented in [9], a complete distance education system involves many functions of the university that we often take for granted, such as registration, a bookstore, and advising. However, online education can be very effective without offering such a complete system. Because we operate in a major urban area and have many working students, our system can best serve regional students who come to campus on rare occasions when they need services or to take exams. This approach is further reflected in our approach to distance teaching, which we call "simulteaching." We use the same software to teach a group of students in the classroom in addition to a number of students over the network at the same time. Classes are recorded as they are sent over the Internet and made available on a server for later playback. The students can and do switch among classroom, Internet live, and Internet playback modes from week to week. Many of the most frequent users of 


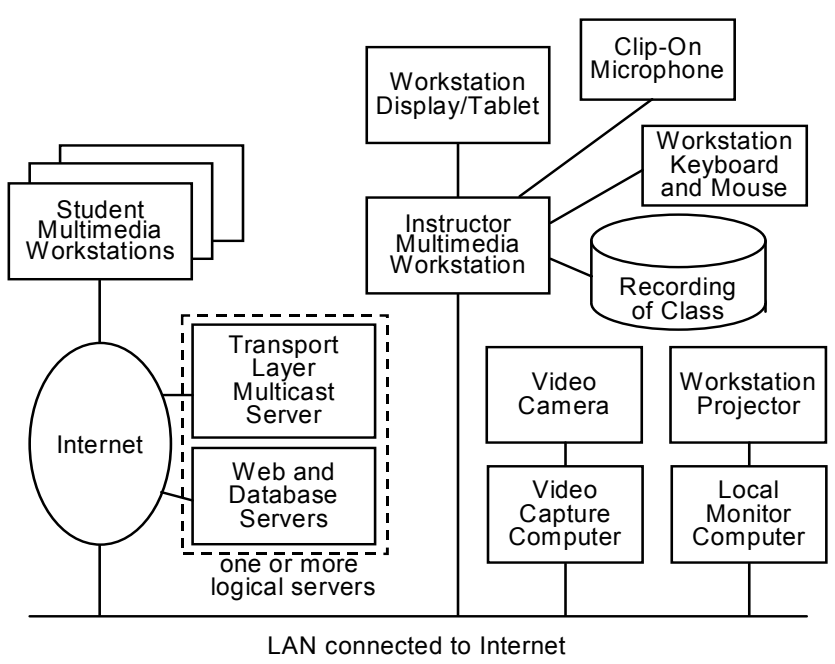

Figure 1. Simulteaching System

the recorded classes are in fact in-person students who have missed a class. Figure 1 shows the system arrangement we use with NEW. Because the classroom projector is driven by the same software used by online students, the whole simulteaching audience receives an identical audiographic presentation.

The NEW system is being used in academic year 20032004 to present 24 courses taught by 14 faculty members in the disciplines of Computer Science and Nursing at George Mason University. Just four years ago only one Computer Science course was taught synchronously, by Pullen. As the workload of NEW has grown, we have been challenged to find ways to scale its support so that we were not overwhelmed by details associated with the growing number of students who use the system for online learning. A critically important innovation in this regard was the NEW Web Portal, which provides access to all functions of the system. The portal simplifies access for users and thereby reduces the amount of support time they must invest to use the system, while at the same time it reduces the amount of staff time needed to support them [10]. In the remainder of this paper we present an overview of the NEW system, followed by a more detailed description of the portal and the role it plays in cost-effective delivery of classes over the Internet.

\section{The NEW Software System}

NEW currently consists of thirteen highly modular opensource software components (Figure 2). Currently it works only under the Windows operating system; however, we have a project underway to expand it to Linux and Macintosh OSX.

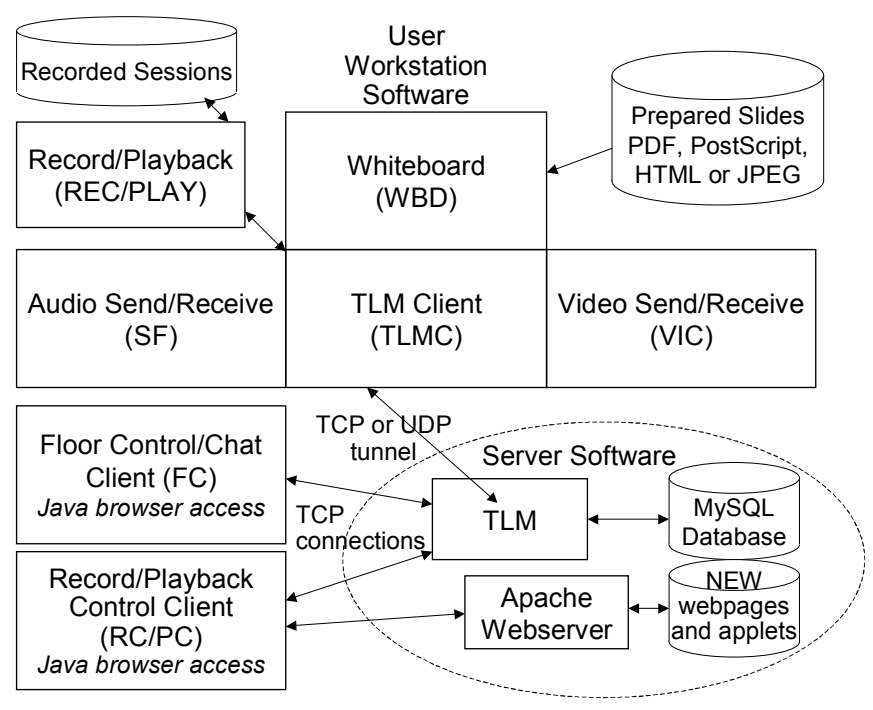

Figure 2. NEW System Components and Interconnection

\subsection{Client Software}

Master Client (TLMC): The multimedia components of NEW were developed originally for use in the Internet multicast environment [11]. The Master Client creates a substitute multicasting environment over a regular network connection, provides TCP tunnels to the live server (TLM), and provides prioritization for data flows from the various client multimedia interfaces. The particular collection of clients and their network configuration established by TLMC is controlled by an Extensible Markup Language (XML) file that normally is downloaded from the supporting webserver at the beginning of a NEW session. TLMC and TLM, along with Floor Control and Record/Playback, are GMU's major contributions to the NEW software made accessible by the portal.

Audio (SF): This component is arguably the most important in the system, because it is essential to the students' experience and also because conveying voice with good quality over the Internet at low data rate presents a big challenge. Speak Freely (SF), is capable of passing good voice quality over the Internet, using a standard sound interface, and requiring only 20 kilobits per second of network capacity. We have added a graphic interface that provides all needed user functions in one easy-to-use package (Figure 3).

Whiteboard (WBD): The other key element for teaching online is graphics. This component provides a shared graphic presentation medium that will display a precomposed graphic prepared in several open formats, most importantly, Adobe Portable Document Format (PDF). These can be annotated with lines, shapes, and text in any color, a very useful feature for maintaining the attention of the visual learner. We prefer to use WBD with an LCD tablet interface so it becomes a surrogate chalkboard. 


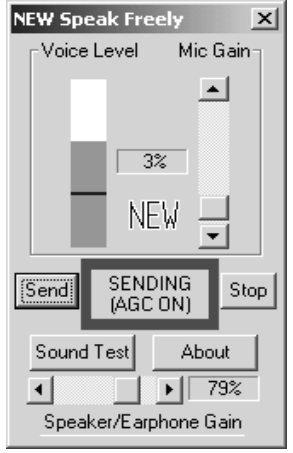

Figure 3. SF User Interface

Video (VIC): We list video after the whiteboard because video is optional in our system, while the WBD is absolutely required. NEW uses an excellent video tool called VIC that is compatible with standard H.323. A typical delivery rate is two frames of 320 by 240 pixels per second, but rates up to 30 frames per second are possible.

Floor Control (FC): This component shows the participants in the session, controls access to the virtual classroom "floor," provides for text questions to the instructor and text chat among the participants, and accepts URLs from the floor holder for browser launch on all participating client systems.

Record (REC) and Record Control (RC): The recorder captures a timestamped stream of messages as seen at a particular user's workstation and records them to disk for playback. RC provides a VCR-like start/stop/pause interface for REC with a display of status and a record counter. REC and RC can run on any Internet-connected computer; they need not be on the same computer with each other or with any particular user.

Playback (PLAY) and Playback Control (PC): The player regenerates the original stream of messages from a timestamped recording. It can function as a playback tool for one user or as a server, streaming playback to multiple users. In either case the same clients (TLMC, SF, WBD, and optionally VIC) provide the user interface.

\subsection{Server Software}

Transport Layer Multicaster (TLM): This component implements the multicast paradigm over the general Internet among a group of participating workstations. It provides access control using passwords and optionally using network addresses checked against the MySQL database. It runs on UNIX or Windows systems.

Playback (PLAY): As noted above, PLAY can be used as a streaming server or run standalone as a user playback tool.
Webserver (Apache): NEW was designed for the Web. We use the Apache open source webserver.

Database (MySQL): We use the open source database access system MySQL which accepts queries in the standard Structured Query Language (SQL). The database provides a unified approach to data management and security across the NEW system and makes possible data access over the network that we are currently using to implement the chat room feature.

\section{Functions of the NEW Web Portal}

While it is possible to run NEW live and playback servers without a Web portal, the mass of detail involved, if more than one course is supported, becomes impractical without automated course management. Therefore we have developed a collection of webpages, programmed in the PHP language, JavaScript, and HTML, that provide the user interface to all aspects of the system.

PHP, which runs on the Apache web server, is a simple but powerful tool for creating HTML content that will run on all major operating systems and web servers. The NEW web portal uses PHP primarily for scripting web server functions and for accessing the MySQL database. In this way, PHP enables the dynamic generation of webpages based on database searches and data passed as session variables from one page to the next but unseen by the user.

\subsection{Teaching and learning functions}

Figures 4 and 5 show the student and instructor interfaces in the portal Welcome page. They are described below.

Software download: the NEW client is installed via the webpage and automatically goes back to the same webpage for self-updating as needed. The installation process was developed to be as simple as possible for the user, and to use only Web browser functions so it would work on all Windows systems.

Student and Instructor live class interface: The student version is available with/without an audio input interface and with/without video. The instructor interface has all of the student functions plus a recording option. Identical software is downloaded to all users; roles and privileges are set in the database and implemented via XML configuration files provided at runtime by the Web server.

Recorded class playback interface: Streaming playback and file download for offline playback both are supported. Access to printable copies of teaching slides also is available. 


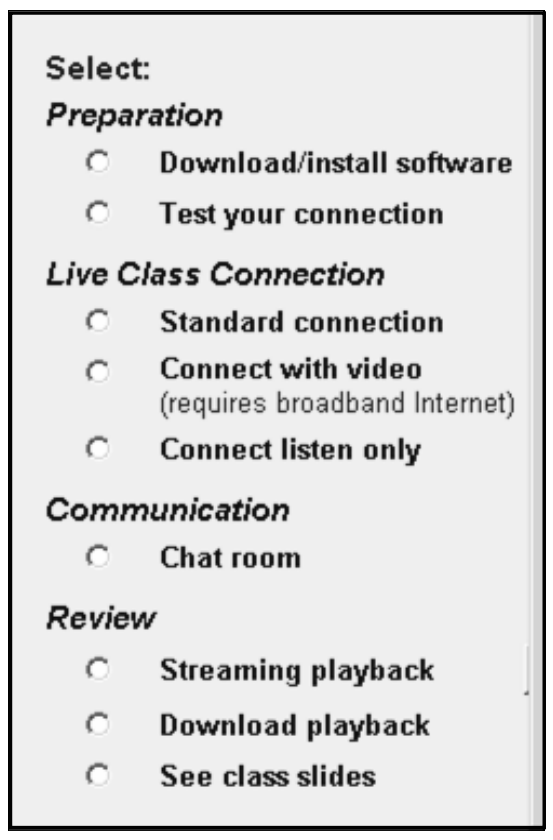

Figure 4. NEW portal student menu

Intra-course communications: Each course has a chat room, implemented using the open source package phpMyChat. These are available 24 hours per day, 7 days per week for meetings of the students with the instructor, teaching assistants, and each other. For staff and faculty there also is an ability to send email to everyone in the database for a particular course.

\subsection{Support and course management functions}

Figure 6 shows the staff and faculty support and management interfaces in the portal Welcome page. The functions are described below.

Slide upload/download: Our staff supports conversion of slides as a service to the faculty, who may submit the slides through the Web portal and retrieve them in WBD format later. PDF format slides or other materials for student use also may be uploaded to the server,

Recording upload: Instructors prefer to run REC on the instructor's computer to ensure that a recording is captured even if the network fails. After a recording is completed, it may be uploaded to the server through the portal.

Class status management: The status of all these uploads and downloads is accessible to the staff and faculty through the portal.

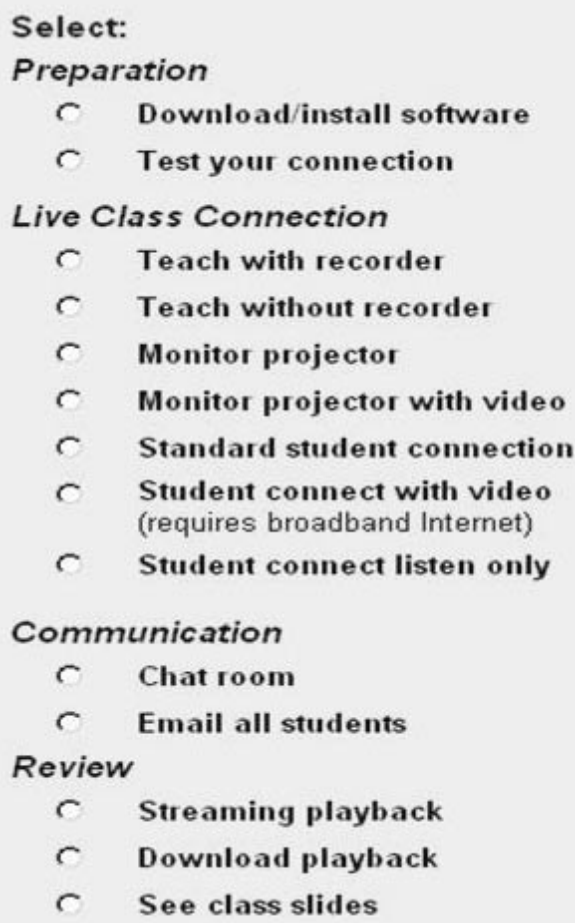

Figure 5. NEW portal instructor menu

Student status management: We build the database using information from the registrar at beginning of the semester. After this, the instructor can update the NEW database to reflect changes to student status and view the class roster.

\subsection{Authentication and Security}

A NEW user logs in via the portal webpage, providing a user ID and a password and choosing from a list of current courses. The user is then authenticated with the database and provided the appropriate NEW menu interface. The user information is passed to NEW pages via Web server session variables enabled by PHP that are unseen by the user. Each NEW page confirms the user's identity by checking the current session variables against the database so that users cannot view any page without successfully logging on (for example, bypassing logon by bookmarking a page or copying a URL).

When a user is authenticated, the time and the client Internet address are recorded and a session identifier is generated. Along with the username and password, these are used to secure the user's entry into the appropriate chat room. If too much time has elapsed since log on, the user must log on again to use the chat room. This precaution is especially important because our students share laboratory computers and may forget to log out. 


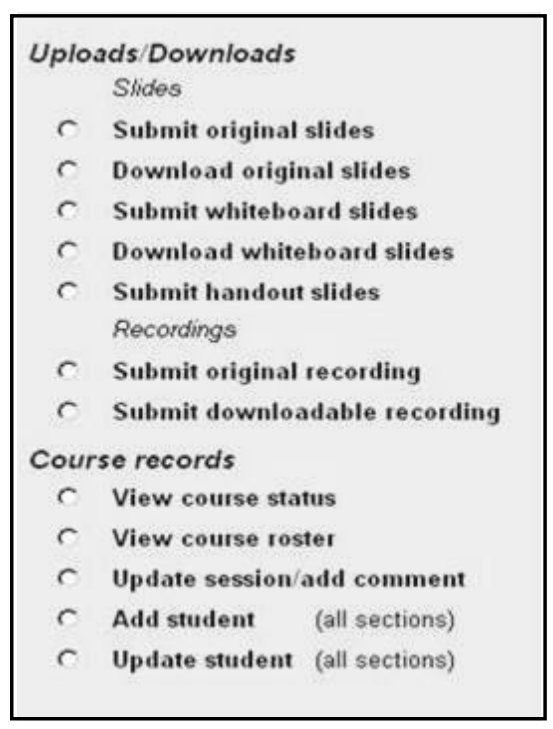

Figure 6: NEW portal staff and faculty menu

\subsection{System administration functions}

Server administration: Staff with appropriate privileges can query the database and make changes, add courses, or start and stop the servers via the portal (Figure 7).

Local options: Because the portal is implemented in webpages, it is easy to add special course information and configuration features by linking in other webpages. This feature also can serve to link in asynchronous learning management systems such as WebCT and Blackboard.

\subsection{NEW information pages}

We also have a complete set of webpages at http://netlab.gmu.edu/NEW, devoted to describing the NEW system development and making the software available in three forms:

- A demonstration portal capability that uses our own server

- A set of download modules for those who want to run a single course on their own servers

- Full access to the source code of NEW for those who want to help continue its development

\section{Conclusions}

We have concluded that a Web-based portal provides an excellent means of supporting a program of multiple synchronous Internet courses. The browser provides an easy, consistent interface for users that can be made
Select request:

$$
\begin{aligned}
& \odot \text { Query NEW database } \\
& \odot \text { Add a course } \\
& \odot \text { Set new semester } \\
& \odot \text { Server status }
\end{aligned}
$$

Figure 7. NEW portal administrator menu

highly intuitive for every system function by effective webpage design. Moreover, the pages can be programmed in conjunction with the NEW database to provide for authentication, logging, and status management. We have chosen to use open source components and post our own work as open source software in order to increase accessibility of Internet distance education to the academic community. Our work on NEW continues; we are working to increase the functionality of the clients and make them available on Linux and Macintosh platforms. We also plan to make the webpages described in this paper available in an easy to install open-source download for organizations that want to scale their use of NEW to multiple courses.

\section{References}

[1] D. Harris, "Online Distance Education in the United States," IEEE Communications, 37(3), 1999

[2] J. Pullen, Synchronous Distance Education and the Internet, Proc. Internet Society Annual Conference, Geneva, Switzerland, 1998, published online at http://www.isoc.org/inet98/proceedings/4b/4b_1.htm

[3] J. Pullen and M. Benson, "ClassWise: Synchronous Internet Desktop Education," multimedia CDROM issue of IEEE Transactions on Education 42(4); printed abstract p. 370

[4] J. Pullen, "The Internet Lecture: Converging Teaching and Technology," ACM Special Interest Group on Computer Science Education (SIGCSE) Bulletin 32(3) 101-104, 2000

[5] J. Pullen, Applicability Of Internet Video In Distance Education For Engineering," Proc. IEEE Frontiers in Education, Reno, NV, 2001, T2F-14-T2F-19, online at http://fie.engrng.pitt.edu/fie2001/papers/1242.pdf

[6] J. Pullen, System Design of Network EducationWare: Open-Source Software for Synchronous Internet Teaching and Learning, Proc. ASEE/SEFI/TUB Colloquium on Technology in Engineering Education, Berlin, Germany, 2002 
[7] J. Pullen, A Software System For Cost-Effective Internet Delivery Of Synchronous Distance Education, Proc. IASTED International Conference on Computers and Advanced Technology in Education 2003, IASTED, Calgary, AB, 2003

[8] J. Pullen and P. McAndrews, Low-Cost Internet Synchronous Distance Education Using Open-Source Software, Proc. ASEE 2004 Annual Conference, American Society of Engineering Educators, 2004, to appear

[9] L. Carswell, The "Virtual University": Toward an Internet Paradigm? ACM SIGCSE Bulletin 30(3), 1998, 46-50

[10] Gery, G., Supporting the Development and Use of Elearning: Designing and Building Portals, tutorial presentation, 19th Annual Conference on Distance Teaching and Learning, Madison, WI, 2003

[11] Macedonia, M. and D. Brutzman, "Mbone Provides Audio and Video Across the Internet," IEEE Computer 27(4), 30-36, 1994 